\title{
The Romanian-Russian Relations and the New Linguistic Hegemony: Towards Natural and Necessary Synergies
}

\author{
lancu Miruna Mădălina \\ PhD, Faculty of History and Philosophy, \\ Babeş-Bolyai University, Cluj-Napoca, Romania
}

Doi: 10.2478/ajis-2018-0030

\begin{abstract}
It is certainly and without fail that through a simple hovering over the present state of relations between Romania and the Russian Federation, the factual balance is not an encouraging one, the state of mind of the bilateral register being continuously dominated by the same issue: ,history is a ballast, and the frame in which the two states perceive each other has been fixed a long time ago, clogged and jammed by momentarily political statements, and deprived of any heuristic nuances" (Dungaciu, Tănăsescu, 2013). Therefore and within such optics, based on an undeniable belief that a heuristic approach of the bilateral dimension is an imperative, the present paper aims to provide a first concrete solution likely to create the critical mass necessary to achieve a complex regeneration process of normalizing Romania's relations with its neighbor to the East. Circumscribed to such a sphere of interest, the present paper assumes the fact that beyond the overwhelming historical legacy related to the bilateral register and implicitly, beyond its problematic receipt, the complex process of normalizing the relations between Romanian and the Russian Federation is unavoidably obstructed by an entire arsenal of terms such as ,, reset”, ,thaw”, ,,recovery”, ,,blocking”, terms which maintain and support a certain negative perception, thus certifying the fact that it prevails a ,freeze”, a ,cooling” and an impediment in the bilateral dimension. Starting from this undeniable and undoubted reality, the axial objective of the present paper is to fundamentally reverse this lexical hegemony, thus operating substantive changes at the level of the linguistic universe specific to the bilateral register, in such a manner as to register a major change in regards to the behavioral pattern afferent to the bilateral reports.
\end{abstract}

Keywords: Romania, Russian Federation, bilateral register, constructivism, linguistic hegemony

\section{Introduction}

Undoubtedly and undeniably, fragmented rather than linear, the Romanian-Russian relation has unraveled and continues to presently unravel, in a sinuous and imprecise manner, with few concrete signals of mutual cooperation, obviously contrasting with the perpetual periods of recoil and suspension. ,If in many Romanian circles there is an aprioristic reluctance towards any format of cooperation with the Russian Federation, for the Russians, Romania is often considered a fascist state, thus remarking, obviously, a two-way conflict relationship in the bilateral plan" (Hărşan, 2007). It prevails in a recurrent dialectics, an ambivalent mistrust, subordinate to a convulsed history, encumbered by light and shadow, responsible for shaping these relations. Integrally, the eventful balance is burdensome. ,It is constantly speaking about pragmatism in the bilateral spectrum, however, the pragmatism evoked cannot eliminate from the Romanian mentality, the image of invasions, wars, deportations, executions, culminating with the repertoire of natural reaction feelings towards an oppressor" (Chifu, 2003). Any early attempt of normalizing the relations is obstructed by the recurrent past in which,,the territorial annexation of Russia/Soviet Union in 1812, and respectively 1940, the seizure of the Romanian treasure and the imposition of 
the communist regime in the postwar period cannot be minimized" (Sava, 2001). In summary, the current state of Romanian-Russian relations is not a gratifying one, the perpetual reference to the past thus leading, with evidence, to the cessation of any constructive steps advanced in the bilateral plan.

Therefore and within such optics, based on an undeniable belief that a heuristic approach of the bilateral dimension is an imperative, the present paper aims to provide a first concrete solution likely to create the critical mass necessary to achieve a complex regeneration process of normalizing Romania's relations with its neighbor to the East. In this regard, the present research attempt places at its nodal analysis center, the premise that a fundamental change and an essential mutation at the level of the lexical construct specific to the bilateral universe can generate substantial transformation of the behavioral pattern of bilateral reports. Accordingly, the novelty element which the present paper proposes lies in sounding the depths of the Romanian-Russian binomial through the perception and the approach lens of the constructivist paradigm, a paradigm specific to the theory of international relations.

Circumscribed to such a sphere of interest, the present paper assumes the fact that beyond the overwhelming historical legacy related to the bilateral register and implicitly, beyond its problematic receipt, the complex process of normalizing the relations between Romanian and the Russian Federation is unavoidably obstructed by an entire arsenal of terms such as ,, resef", ,,thaw”, ,,recovery”, ,blocking”, terms which maintain and support a certain negative perception, thus certifying the fact that it prevails a ,,freeze”, a ,,cooling” and an impediment in the bilateral dimension. Starting from this undeniable and undoubted reality, the axial objective of the present paper is to fundamentally reverse this lexical hegemony, thus operating substantive changes at the level of the linguistic universe specific to the bilateral register, in such a manner as to register a major change in regards to the behavioral pattern afferent to the bilateral reports. Equally it is no less significant the fact that the results and the conclusion, which we engage with temerity to present, aim to provide a wider and substantial panorama afferent to the subject concerned, thus providing at the same time, a concrete landmark in setting out new directions and approach strategies with regards to the issue subjected to the investigative filter.

\section{The Romanian-Russian Immediate Universe. A Synoptic Presentation Afferent to the Bilateral Constellation}

Although it is undisputable that Romania's relations with the Russian Federation are not historically exemplary and do not represent a defining landmark for harmony and good understanding in the register of interstates relations, it is equally true that in the present time the rapports between the two state entities do not take place under the most promising auspices and have reached the point where they can hardly benefit from a profound and far-reaching development. Practically, for almost two and a half decades, the relations between the two states fail to evolve into a natural and inherently normality area and to develop to the desired and expected capacity. The reasons for such a state of spirit are extremely complex and are mainly related to a series of problems specific to the bilateral framework. Thus, a first problem which is responsible for the delay of synergic and lasting horizons in the bilateral dimension is related to the sinous evolution of diplomatic relations and implicitly, ,,to the negative passive acumulated in the space of bilateral relations for almost two and a half decades" (Goşu, 2017).

As a result and under such circumstances, it can be said that shortly after the official disruption of the Soviet Union and the assumption of the main responsibilities by the Russian Federation, there had not been capital mutations and substance realities in the sphere of the bilateral Romanian-Russian relations because ,, at the political level, the relations between the two states had been affected on the long term by the different positions adopted in regards to the evolution of the situation in the Republic of Moldova following the proclamation of its independence and especially after the tragic events that took place in this country in the summer of 1992" (Buga, 2003). Equally, the relations did not evolve on new and pragmatic coordinates, coordinates truelly eliberated from the ballast accumulated along the common past, because all the Romanian decision-making institutions focused exclusively on the achievement of the two major objectives of 
integration into NATO and EU structures, situation that led to the exit of the Russian Federation from the spheres of interest and priorities of the romanian state foreign policy. In addition, ,,the improper atmosphere installed in the bilateral plan was further deepened in April 1996, when the former head of the Russian diplomacy, Evghenii Primakov arrived in Buchared and was put in the impossibility to paraph the new Romanian-Russian basic political treaty, as a result of some objections communicated in the last moment by the Romanian side"( Buga, 2003). Naturally and somewhow predictable, this episode has been accounted in the bilateral dimension and significally imprinted the subsequent trajectory of relations, matter for which during the 1997-2000 period there were no changes of substance and significant advances in the sfere of bilateral relations, thus prevailing an extremely complicated and controversial dialogue in the bilateral dimension.

However, ,, after 2000, the substantive dialogue with Russia came to the attention of the Romanian diplomacy and resulted in the signing in July 2003 of the Romanian-Russian basic political treaty ratified by the Parliaments of the two countries" (Hărşan, 2007). Despite all this and implicitly, despite this gratifying and gladdening reality, the treaty failed to confer a new dimension to the bilateral constellation, and failed to lead to a spectacular development of relations and to abrupt and forward-looking advances in the bilateral segment. In addition, ,,in 2005-2007 period but also later on, the Romanian-Russian bilateral relation gained new features, respectively became unpredictable and full of conflict, because instead of a natural and normal evolution in the sphere of the bilateral rapports, followed a bellicose rhetoric in the bilateral plan" (Hărşan, 2007), a rhetoric which has unavoidable affected the trajectory of the relations. At the same time, over the past three years, the complicated dossier related to the Romanian-Russian bilateral rapports, has been too little visible on the foreign policy agenda of the two countries, so, in such conditions, it can be advanced the idea that currently, the subject of the Romanian-Russian relations is extremely peripheral, a situation that explains to a great extent why the relevant and content dialogue in the bilateral area has become more and more a deserted reality and the high-level meetings between the two countries are beginning to become also, with celerity, ,,chapters of history".

Beyond this aspect however, it is equally true that until the present moment the RomanianRussian bilateral relations could not ignore also the historical ambiance consubstantial to the bilateral universe, rearranging and reconfiguring itself in relation to the burdensome dowry inherited in the bilateral plan. Thus, for more than twenty-five years, the major touchstone afferent to the bilateral framework continues to remain the dimension of a sinuous history, with its highly problematic and delicate valences, respectively:

"the territorial controversy ( a big part of the Republic of Moldova but also parts of Ukraine, at which passed the territory of Bessarabia and Northern Bukovina after the 1940 annexation; the Romanian treasure filed in Moscow in 1916-1971 and not entirely recovered; the moral satisfaction by the conviction of the Ribbentrop-Molotov Pact (1939)" (Goşu, 2017). Naturally and unquestionable regarding this problematic issues with historical substratum "nothing has changed and everything seems frozen in the project" (Dungaciu, Tănăsescu, 2013), a situation which is acutely felt in the bilateral sphere and only delays and especially prevents the perspective of profitable, continuos and content relations in the theater of bilateral arrangements.

In addition, beyond the perrenial aspects consubstantial to the bilateral historic, until the present moment, the Romanian-Russian bilateral agenda has added a further set of burning subjects and topics (the Transnistrian problem, the opposition of the Russian Federation towards the integration of Romania in the North-Atlantic structures, the problem of the Black Sea region, the establishment of the American military bases on the territory of the Romanian state, the placement of the American missile shield from Deveselu) topics which, otherwise, don't do but to complicate in a considerable manner the evolution and the devlopment of the bilteral tandem. At the same time, it is equally relevant the fact that in the Romanian-Russian bilateral dimension one can also identify a certain form of two-way adversity, result of a poor, critical and defective perceptual culture at both sides, a state that most often serves to wrong and counterproductive decisions in the bilateral plan.

Consequently and under such circumstances, the overall image that emerges from this synoptic presentation of the bilateral trajectory is the one that highlights the fact that currently the relations between Romania and the Russian Federation do not evolve under the best auspices and this situation is due primary to the sinuous diplomatic path, the historical meander and the 
additional frictions, and implicitly to the two-way state of adversity.

\section{The Romanian-Russian Bilateral Relations and the Need for a New Linguistic Hegemony}

Ultimately, given the fact that the Romanian-Russian bilateral register distinguishes itself by an extremely burdensome diplomatic, historical and perceptual ambiance, there is an urgent need for a new optics strategy but also for an heuristic approach related to the bilateral dimension, respectively an approach that is susceptible to eliminate the deficit existent in the bilateral plan. In this regard, such an approach can be the one that starts from the premise that an essential change and a substance mutation at the lexical construct specific to the bilateral register can generate substantive transformation afferent to the behavioral pattern of bilateral relations, the basis of this approach being thus, an in-depth survey of the Romanian-Russian binomial from the perception lens of the constructivist paradigm, a paradigm specific to the theory of international relations.

Basically, "the constructivism paradigm is consubstantial to the field of European integration, and can be defined as having at the base the ontology which insists upon the fact that the human agents do not exist independent the social environment and the common system of values shared, broadly defined as culture" ( Popescu, 2009). In other words, the constructivists confer substance to the character rather social than material of the social structures, aspect which means that any perspective is designed by people and is not predetermined from outside. "The states, the societies and the individuals are not led and ruled by immutable forces, but rather by a widely shared knowledge which creates a way or other to get in touch. This culture is built over in time and through continuous interactions at all levels, being subjected to permanent developments. Change is a social condition and its engine are ideas" (Leucea, 2012). Equally, the constructivists highlight the fact that language structures our thinking mechanism, our cognitive life. "Certain words, a certain terminology and implicitly, a specific language used, shows the way in which we think and we legitimize various actions, the way in which we interpret an event, a political action or a certain behavior" (Leucea, 2012).

Therefore and under such circumstances, the language used is extremely important because it confers weight to the action undertaken, constructing or deconstructing substance realities in various spheres of interest and in different fields of activities. Practically, the language and implicitly its ultimate relay, the word "is an extension of the thought or simply a certification or an attestation of the thought. When a word is spoken, a contract with the quantum field is signed which means that this is how it has to be, and consequently it is agreed that everything should be carried out according to the observer's intention" (Danc, 2017). The word is therefore a living force, susceptible of inducing essential mutation in the palpable dimension, matter for which there is an urgent need to pay considerable attention to the way in which it is shaped and structured this indisputable and incontestable reality. In this sense, it is very important to appeal to creative words, words that are concrete vectors of action and to eliminate the negative language, language which blocks the materialization of any approach.

From this point of view, the Romanian-Russian bilateral register is an extremely conclusive example of interstate rapport at the level of which it prevails an implanted and pre-established language, with totally inadequate and defective terms, language that gives measure and confers form to these relations, structuring the evolution of the bilateral trajectory. Practically, at a simple ascertaing overflight afferent to the lexical construct specific to the bilateral constellation, one can easily notice the fact that it exists an entire arsenal of termes not exacly suitable and proper (,,reset”, ,thaw”, ,,recovery”, ,,blocking”, ,vitiated”, ,failure”, ,, negative trend”, ,,deadlock”, ",regression"), terms which construct o negative reality in the bilateral dimension and support a state improper for the natural and normal development of these rapports. Such terms, recurrent encountered in the universe of bilateral relations only confer weight to such a state of spirit, thus certifying that ist exists a frost and an impediment in the Romanian-Russian bilateral dimension.

Starting from this undisputable reality, the Romanian-Russian bilateral relations need a fundamental change in the dimension of the manner in which they construct their own narrative, so implicitly they need a mutation transformation at the level of the linguistic ethos, the language which 
structures and shapes their universe. It is therefore necessary to carefully select a register of creative terms such as ,pozitive dynamics”, ,,abrupt advance”, ,synergy”, ,convergence” ,,collaboration”, ,,solid base”, ,friendship", ,good-understanding”, ,, harmony”, and implicitly to abandon the current linguistic mechanisms, that generates inadequate realities in the bilateral plan. In other word, there is an urgent need for a new linguistic hegemony in the sphere of bilateral relations, a hegemony susceptible of inducing substatial mutations in the concrete dimension, thus constructing essential realities in the bilateral plan and structuring adequately and properly the course of these relations. Ultimately, there is a need for a new linguistic order, an order creative of natural and full of substance horizons, as an optimal solution for bringing things in the bed of the inherent normality!

\section{Conclusions}

Undoubtedly and indisputably, the current state of the Romanian-Russian bilateral relations calls for an urgent need of change in the bilateral plan and implictly, calls for a profound optics mutation in the way and the manner of approaching these bilateral dimension. In this sense, it is imperative to fundamentally reevaluate and rethink the linguistic scaffolding and structure specific to the bilateral construct, ranging from the current linguistic order, an order which generates totally inadequate and inappropiate realities in the bilateral plan to a new linguistic hegemony carefully and responsable configured, hegemony that by its inherent force is truelly able to dimension a natural and synergic universe in the Romanian-Russian bilateral sphere and to mark a substance chage in the dimension of these vital and capital relations for both states!

\section{References}

Buga, V.(2003), Romanian-Russian relations: stage and perspectives (pp.10-11), Occasional papers: House NATO;

Chifu, I.(2003), The long way from dialogue to cooperation (p.27), Occasional papers: House NATO;

Dungaciu, D., Tănăsescu, G. (2007) Romania and Russia after 20 years: Perceptions, Realities, Perspectives (p.5.), Bucharest: Institute of Political Sciences and International Relations;

Danc, R. (2017), How we create our reality, [Available] Online: https://astrocercetas.wordpress.com /2017/06/02/cum-ne-cream-realitatea/ ;

Goşu, A. (2017), Scenarios in the Romanian Russian relations. Magazine 22, [Available] Online: http://revista22online.ro/70260207/scenarii-n-relaiile-romno-ruse.html;

Hărşan, C. (2007), Romania-Russia where to? (III). Free Romania, [Available] Online: http://romanialibera.ro /special/documentare/romania-rusia-incotro---iii--112277;

Idem, (2007), Romania-Russia where to? (IV). Free Romania, [Available] Online: http://romanialibera.ro /special/documentare/romania-rusia--incotro---iv--112324.

Idem, (2007), Romania-Russia where to? (V). Free Romania, [Available] Online: http://romanialibera.ro /special/documentare/romania-rusia--incotro---v--112431;

Leucea, I, (2012), Constructivism and human security, laşi: European Institute;

Popescu, E, (2009), Theories of European integration (p.33), Bucharest: C.H.Beck,

Sava, N.I., (2001), Romanian-Russian Relations in the Context of the Euro-Atlantic Integration Process. Conflict Studies, [Available] Online:

http://www.isn.ethz.ch/isn/Digital-Library/Publications/Detail/?ots591=0c54e3b3-1e9c-be1e-2c24a6a8c7060233\&lng=en\&id=97570 . 